Mens

revue d'histoire intellectuelle de l'Amérique française

Gilles Paquet et Jean-Pierre Wallot. Un Québec moderne 1760-1840. Essai d'histoire économique et sociale. Montréal, Éditions Hurtubise HMH, 2007. 735 p.

\title{
Marcel Bellavance
}

Volume 9, numéro 2, printemps 2009

URI : https://id.erudit.org/iderudit/1023105ar

DOI : https://doi.org/10.7202/1023105ar

Aller au sommaire du numéro

Éditeur(s)

Centre de recherche en civilisation canadienne-française

ISSN

1492-8647 (imprimé)

1927-9299 (numérique)

Découvrir la revue

Citer ce compte rendu

Bellavance, M. (2009). Compte rendu de [Gilles Paquet et Jean-Pierre Wallot. Un Québec moderne 1760-1840. Essai d'histoire économique et sociale. Montréal, Éditions Hurtubise HMH, 2007. 735 p.] Mens, 9(2), 310-314.

https://doi.org/10.7202/1023105ar d'utilisation que vous pouvez consulter en ligne.

https://apropos.erudit.org/fr/usagers/politique-dutilisation/ 
au pamphlétaire maudit. C'est ça, la révolution, pas une révolution tranquille, insignifiante, rose nénane, mais une révolution véritable, nécessaire et noble » (p. 70).

Dominique Garand Département d'études littéraires Université du Québec à Montréal

Gilles Paquet et Jean-Pierre Wallot. Un Québec moderne 1760-1840. Essai d'histoire économique et sociale. Montréal, Éditions Hurtubise HMH , 2007. 735 p.

Cet essai réunit onze articles ou chapitres de livre les plus représentatifs de l'œuvre historique de leurs auteurs au cours des trente dernières années. Il s'agit donc de rééditions peu revues de ce que Jean-Pierre Wallot et Gilles Paquet estiment constituer l'essentiel de la réflexion qui fonde largement leur analyse de l'histoire économique et sociale du Québec de 1760 à 1840 et, tout particulièrement, la position moderniste qu'ils ont adoptée dans le débat historiographique.

Toute une génération d'historiens reconnaittra ce débat omniprésent dans les années 1960-1970 et qui perdure encore aujourd'hui avec la publication de cet ouvrage. Deux thèses contradictoires sur le Québec de cette période s'affrontent dans ce débat. L'une « traditionnelle » fondée sur les effets explosifs - la rébellion de 1837 - de la combinaison d'une «crise agricole dès 1802 » et du "nationalisme réactionnaire " "des masses paysannes " canadiennes-françaises " agitées par une élite locale de membres des professions libérales » (p. 278, 297, 362-363, 439-440). C'est la thèse proposée par Fernand Ouellet, principalement dans son Histoire éco- 
nomique et sociale du Québec, 1760-1850. L'autre thèse prétendument moderne est défendue par les présents auteurs et un groupe d'historiens plus jeunes dits « révisionnistes".

Se trouvent donc ainsi en présence deux interprétations incompatibles du passé québécois dont l'une qualifiée aussi de « mythique » fait l'objet d'une « déconstruction » systématique en même temps qu'est énoncée une nouvelle problématique de modernisation de la socio-économie du Québec au tournant du $\mathrm{XIX}^{\mathrm{e}}$ siècle, suggérant ainsi l'amorce de la transition de ce dernier vers le capitalisme industriel comme cela s'est produit dans le monde atlantique (p. 53-61). Cette démarche de construction-déconstruction traverse tous les chapitres ou articles de la seconde partie de ce gros livre (chapitres 5 à 11), intitulée "La seconde grande discontinuité », référence à la problématique des «Quatre grandes discontinuités » préalablement expliquée dans le premier chapitre de la première partie de l'essai.

Le parti pris adopté par les auteurs de publier presque tels quels ces "mélanges » explique, me semble-t-il, les répétitions et le retour récurrent aux mêmes thèmes comme c'est le cas pour la thèse de Ouellet sur la crise agricole et ses répercussions sociales et politiques. Une réédition mieux intégrée aurait-elle pu être envisagée ? Qu'un tel livre soit publié aujourd'hui montre bien cependant à quel point le débat sur l'œuvre de Ouellet fut stimulant pour la recherche en général et pour l'œuvre de Wallot et Paquet en particulier. Les historiens des cycles supérieurs d'études et les lecteurs avisés y trouveront leur compte. Ils disposeront aussi de références à une masse documentaire énorme sur la période, colligée par de nombreux chargés de recherche, et commentée par les auteurs dans les quelques 150 pages de notes et renvois. La lecture concomitante du texte et de tout l'appareil critique et bibliographique rend la fréquentation de cet ouvrage ardue. Marc Bloch, dans son Apologie pour l'bistoire ou métier d'bisto- 
rien, se montrait en son temps visiblement agacé par cette pratique :

Les marges inférieures des pages exercent sur beaucoup d'érudits une attraction qui touche au vertige. Il est sûrement absurde d'en encombrer les blancs, comme ils le font, de renvois bibliographiques qu'une liste, dressée en tête du volume, eût, pour la plupart épargnés; ou pis encore, d'y reléguer, par pure paresse, de longs développements dont la place était marquée dans le corps même de l'exposé; en sorte que le plus utile de ces ouvrages, c'est souvent dans la cave qu'il le faut chercher. (p. 40)

Abordons maintenant un aspect important de l'argumentaire moderniste dont se réclament Wallot et Paquet, et qui a été plutôt effleuré dans leur essai. C'est la corrélation existante selon les théoriciens de la nation et du nationalisme comme Ernest Gellner, Karl Deutsch, Antony Smith, Myrolav

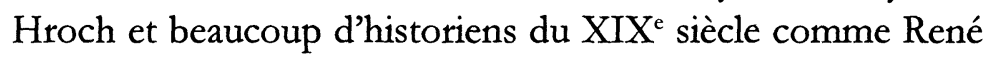
Girault, René Rémond, Patrick Cabanel, Nicolas Rousselier et Eric Hobsbawm entre courant libéral et courant national, entre modernisation de la socio-économie et nationalisation des sociétés et, enfin, entre ces phénomènes et le rôle crucial joué par les classes moyennes, en particulier les membres des professions libérales. Nulle part, nous semble-t-il, trouve-ton dans le présent essai, malgré les nombreuses pages de notes et de renvois, référence à ces auteurs reconnus. Ceci s'explique du fait que les modernistes, sur ce plan, rejoignent les historiens traditionnels ou conservateurs dans la dissociation des notions de libéralisme, de nationalisme et de modernité.

Siècle du capital, siècle du libéralisme, siècle des nationalités, autant de vocables récurrents pour définir le XIX $\mathrm{X}^{\mathrm{e}}$ siècle dont la complémentarité ne semble pas avoir intéressé Wallot et Paquet. Ces derniers s'approchent pourtant de très près de cette perspective globale quand ils écrivent que le 
nationalisme n'a pu être engendré par la seule crise agricole mais par un ensemble de conflits et de discordes qu'ils ont schématisés dans les tableaux 5.1 (p. 294) et 9.1 (p. 550) intitulés Conflits et discordes. Superposition de conflits aux niveaux " constitutionnel, social, ethnique et économique » qui est au cœur de leur " cosmologie de rechange ». C'est, disent-ils, ce qui fait «la particularité du cas bas-canadien» (p. 551). Ne fallait-il pas nuancer cette interprétation et affirmer que la lutte pour la responsabilité ministérielle au Bas-Canada était davantage politique que constitutionnelle? C'est ce qui distinguerait, pensons-nous, le Bas-Canada du Haut-Canada. Dans le même ordre d'idées, l'opposition ente les Britanniques au pouvoir et les Canadiens n'est-elle pas davantage nationale qu'ethnique?

La démarche de Wallot et Paquet ainsi nuancée se rapprocherait de celle d'Ernest Gellner pour qui une situation n'est vraiment nationaliste que lorsque deux cultures vivent sur le même territoire et que l'une d'elle possède le pouvoir du seul fait de son appartenance culturelle. C'est « le peuple à l'anglaise » par opposition « au vrai peuple », c'est-à-dire aux Canadiens (p. 550-551).

L'ambiguité dans l'analyse des niveaux de conflits s'aggrave avec l'utilisation imprécise et anachronique du vocabulaire relatif aux Canadiens et aux Britanniques. Les premiers sont déjà relégués au statut de Canadiens français tandis que les seconds sont délestés de leurs attributs impérialistes, colonisateurs ou conquérants pour se métamorphoser tantôt en Canadiens anglais tantôt en nos compatriotes anglophones (p. 295, 300, 408, etc.).

En avant-propos, Wallot et Paquet soulignent avec raison l'importance des données quantitatives «pour déraciner les préjugés ", les "simplifications que permettent les périodes proto-statistiques » (p. 22). Il convient donc de souligner particulièrement la cueillette systématique de données pour 
étayer leurs interprétations. On peut facilement s'en convaincre à la vue des quelques 70 tableaux et graphiques qui parsèment l'ouvrage.

En général, ce sont des tableaux à double entrée où sont compilées des données brutes (les tableaux 6.1 à 6.4 par exemple, p. 317-323) qui nous informent sur l'évolution d'un phénomène au fil des ans. Ces tableaux sont ordinairement informatifs. Ils sont en effet rarement traités. Dans l'exemple choisi pour les fins de la démonstration, il s'agit du « nombre et [du] tonnage des navires à l'entrée et à la sortie du port de Québec ». Aucun traitement de l'information (pourcentage, totaux, etc.) n'est fait, si bien qu'aucune perception globale du tableau n'est accessible au lecteur. Mais dans les interprétations qui sont faites, les auteurs parlent de moyenne et de pourcentage souvent approximatifs qui nous laissent à penser qu'il y a eu un début de traitement dont les résultats apparaissent dans le commentaire seulement. Il en est ainsi « des quatre étapes » depuis le début de la période étudiée et des moyennes dont on chercherait en vain la trace dans le tableau 6.1 (p. 316) et du tableau 8.1 où il y a peu de correspondances entre les données du tableau et celles du commentaire (p. 452). Il s'agit en fait de données que le lecteur doit calculer lui-même comme c'est le cas encore pour le tableau 8.2 (p. 459) qui est " une ventilation en pourcentage " sans qu'il y ait le rapport à 100. Cela dit, il reste qu'un meilleur traitement de l'information aurait permis aux auteurs de communiquer plus clairement les résultats d'une recherche par ailleurs fort imposante. Il faut aussi souligner les qualités de démonstration de leur problématique d'un Québec «entrant pleinement dans la modernité » au tournant du $\mathrm{XIX}^{\mathrm{e}}$ siècle.

Marcel Bellavance

Division des Études permanentes Collège militaire royal du Canada 